\title{
Dispersión polínica y éxito reproductivo de cuatro especies arbóreas de un bosque xerófito de Argentina
}

\author{
Juan Pablo Torretta ${ }^{1} \&$ Alicia M. Basilio ${ }^{2}$ \\ 1. Cátedra de Botánica Agrícola, Facultad de Agronomía, Universidad de Buenos Aires Av. San Martín 4453 - \\ C1417DSE - Buenos Aires - Argentina; torretta@agro.uba.ar \\ 2. Apicultura, Facultad de Agronomía, Universidad de Buenos Aires Av. San Martín 4453 - C1417DSE - Buenos Aires \\ -Argentina; abasilio@agro.uba.ar
}

Recibido 27-VI-2007. Corregido 25-VI-2008. Aceptado 29-VII-2008.

\begin{abstract}
Pollen dispersion and reproductive success of four tree species of a xerophytic forest from Argentina. The "talares" in eastern Buenos Aires province, Argentina, are coastal xerophitic forests structured by few arboreal species surrounded by a lower and moister soil matrix. We studied the reproductive parameters of the most representative arboreal species (Celtis tala, Scutia buxifolia, Jodina rhombifolia, and Schinus longifolia). Pollen dispersion was studied through floral visitor traps (biotic dispersion) and using gravimetric pollen collectors (abiotic dispersion). The reproductive success (fruit formation rate) of the focal species was studied by enclosing flowers with different mesh bags. The reproductive system varied among the different species. C. tala was anemophilous and selfcompatible. S. buxifolia was entomophilous and floral visitors dependant. $J$. rhombifolia was entomophylous, although spontaneous autogamy could favor reproduction in the absence of pollinators. Lastly, S. longifolia could be an ambophilous species (pollinated by insects and by the wind). This dual system may be the result of system flexibility mechanism or an evolutionary transition. Rev. Biol. Trop. 57 (1-2): 283-292. Epub 2009 June 30.
\end{abstract}

Key words: pollen dispersion, trees, pollination, ambophily, anemophily, entomophily, xeric forest.

Los bosques de tala y coronillo, denominados "talares", del este de la provincia de Buenos Aires (Argentina) se ubican sobre cordones de conchilla fósiles subparalelos al Río de la Plata (Ribichich 1996, Stupino et al. 2004), cuyas características edáficas les confieren mayor xericidad con respecto a los suelos que los rodean (Murriello et al. 1993). Los cordones están inmersos en una matriz de suelos más bajos y húmedos dominados por numerosas especies de ciperáceas y gramíneas (Parodi 1940, Vervoorst 1967). Las especies arbóreas del bosque están relacionadas con las del Bosque Chaqueño, que se desarrolla unos $1000 \mathrm{~km}$ al $\mathrm{N}$, y estos bosques constituyen los únicos del distrito pampeano (Parodi 1940, Vervoorst 1967).

Habitan seis especies arbóreas nativas, Celtis tala Gill. ex Planch, "tala", Scutia buxifolia Reiss, "coronillo", Jodina rhombifolia (Hook. et Arn.) Reiss, "sombra de toro", Schinus longifolia (Lindl.) Speg., "molle", Sambucus astralis Cham. \& Schltdl. "sauco" y Phytolacca dioica L. "ombú", que constituyen la dominancia estructural de la comunidad, acompañadas por numerosas especies herbáceas y arbustivas (Arturi et al. 2006, Basilio y Torretta 2006). Algunas de estas especies arbóreas alcanzan su límite austral de distribución en estos bosques extrazonales. Estos árboles carecen de propagación vegetativa, por eso la polinización y la producción de frutos y semillas viables son imprescindibles para su perpetuación y el mantenimiento del bosque.

Las plantas con flores exhiben diversos mecanismos de dispersión polínica y sistemas reproductivos. La morfología de las flores, la 
recompensa ofrecida y la fenología de floración de las distintas especies vegetales muchas veces están asociadas al tipo de dispersión polínica, lo cual ha llevado a diversos autores a la descripción de síndromes de polinización (Faegri \& van der Pijl 1979, Proctor et al. 1996). Asimismo, el sistema reproductivo, la morfología de las flores y del polen y la fenología de floración están asociados al tipo de dispersión polínica. Las plantas anemófilas se caracterizan por tener flores unisexuales, anteras de gran tamaño o mayor número de flores masculinas, alta relación polen/óvulo, polen seco, pequeño y liso (Endress 1998). Actualmente, la anemofilia es considerada una condición derivada en angiospermas que se ha originado repetidamente en diversos linajes de angiospermas (Culley et al. 2002). Es predominante en ambientes secos y abiertos, tales como pastizales y sabanas, aunque también está presente en especies arbóreas de bosques. Bush \& Rivera (2001) comentan que en bosques templados, el 70-100\% de las especies arbóreas son anemófilas mientras que en bosque tropicales sólo alcanzan el 2-3\%. Por otro lado, las especies entomófilas se caracterizan por tener flores de colores llamativos, fragantes, con recompensas (principalmente néctar y polen), granos de polen con ornamentaciones (a menudo con pollen-kitt) y una relación polen/óvulo moderada (Endress 1998). La entomofilia está presente en todos los ambientes y es el más común de los sistemas de dispersión polínica. La ambofilia, es decir, la polinización por algunos insectos y el viento, simultáneamente o secuencialmente dentro de la estación de floración, es un fenómeno común (Bullock 1994) y antiguamente mencionado por Kerner von Marilaun en 1895 (cita en Bullock 1994). Culley et al. (2002) mencionan a la ambofilia como un posible estado transicional entre la entomofilia y la anemofilia y la posibilidad de que la ambofilia sea un importante sistema de polinización abiótico generalista en plantas.

La expansión de la frontera agrícola y el cambio en el uso de la tierra amenaza la supervivencia y estabilidad de los talares del E de la provincia de Buenos Aires. La alteración del bosque comenzó con la tala selectiva de sus árboles para leña, las actividades ganaderas también causaron deterioros en la comunidad así como la extracción de conchilla, con desmote total del bosque (Parodi 1940, Vervoorst 1967, Goya et al. 1992, Chichizola 1993, Morello 2006). Si bien existen algunos sectores donde el bosque ha sido protegido, en general estos talares presentan distintos grados de degradación y han sido colonizado por especies arbóreas exóticas fuertemente invasoras como Ligustrum lucidum Ait., L. sinense Lour. y Gleditsia triacanthos L. (Ruiz Selmo 2003). La comprensión de los aspectos de la reproducción de las especies que dominan la estructura arbórea de esta comunidad posibilitaría trazar planes de manejo para su conservación. Por ello, el objetivo de este trabajo es determinar el sistema de dispersión polínica y la tasa de producción de frutos de las cuatro especies arbóreas nativas más abundantes.

\section{MATERIALES Y MÉTODOS}

Sitio de estudio: el trabajo se desarrolló en el campo Carretero, ubicado dentro de la Reserva de Biosfera MAB-UNESCO Parque Costero del Sur (entre los $35^{\circ} \mathrm{S}, 57^{\circ} 30^{\prime} \mathrm{W}$ y $35^{\circ} 45^{\prime} \mathrm{S}, 57^{\circ} 15^{\prime} \mathrm{W}$ ), en el Partido de Magdalena, Provincia de Buenos Aires. Las visitas al campo se realizaron quincenalmente, entre mayo de 1999 y febrero del 2000.

Especies estudiadas: el estudio se realizó sobre las cuatro especies arbóreas nativas más abundantes del talar: Celtis tala Gill. ex Planch., Scutia buxifolia Reiss, Jodina rhombifolia (Hook. et Arn.) Reiss y Schinus longifolia (Lindl.) Speg.

Celtis tala (Celtidaceae), es un árbol espinoso, caducifolio, con inconspicuas flores hermafroditas y masculinas (Hunziker y Dottori 1976). El polen es triporado, con depresiones o utrículos en la endoapertura, isopolar, esferoidal y con exina lisa a escarbada de 25-29 $\mu \mathrm{m}$ de diámetro. Florece durante la primavera y la floración coincide con la aparición de las primeras hojas (Murrielo et al. 1993). Constituye 
una especie común en bosques xerófilos de Argentina alcanzando su distribución hasta el NE de la provincia de Buenos Aires (Parodi 1940).

Scutia buxifolia Reiss (Rhamnaceae), es un árbol perennifolio, con ramas espinosas y de flores pequeñas, hermafroditas y nectaríferas dispuestas en fascículos paucifloros. Los granos de polen son esféricos a sub-esféricos, tricolpoaperturados con anulus, de exina reticulada a lisa, de unos 30-35 $\mu \mathrm{m}$ de diámetro. Su distribución comprende desde Bolivia, Brasil, Paraguay, Uruguay y norte de Argentina hasta los bosques xerófitos del $\mathrm{E}$ de la provincia de Buenos Aires (Johnston 1974, Tortosa, 1995). Junto a $C$. tala, estas especies representan el 90\% de los parámetros estructurales de estos talares (Goya et al. 1992).

Jodina rhombifolia (Santalaceae), es un árbol perennifolio, con hojas coriáceas, romboidales, terminadas en espinas. La floración es invernal (Murrielo et al. 1993) y las flores son hermafroditas, amarillentas pálidas y nectaríferas, reunidas en glomérulos axilares (Ulibarri 1987). Los granos de polen son esféricos a sub-esféricos, lisos y pequeños (20 a $25 \mu \mathrm{m}$ de diámetro) tricolpoaperturados y de contorno triangular. Su distribución es amplia, encontrándose en Bolivia, Paraguay, Brasil, Uruguay y Argentina. En Argentina se la halla desde Chaco hasta Río Negro y es común en los bosques xerófilos y ribereños del Río de la Plata (Ulibarri 1987).

Schinus longifolia (Anacardiaceae), arbolito de follaje perenne y ramas espinescentes, sus flores son unisexuales, las masculinas poseen 10 estambres didínamos y gineceo vestigial, las femeninas presentan los estambres reducidos y el ovario globoso, con 3 estilos concrescentes y estigmas capitados (Muñoz 1990). Los granos de polen son tricolporados, esferoidales a subprolados, con la exina estratificada, estriada, de $27-30 \mu \mathrm{m}$ de diámetro. Su distribución es amplia, desde Brasil, Paraguay, Uruguay y este de Argentina hasta los bosques del Río de la Plata (Fabris 1965).

La nomenclatura botánica usada en el trabajo sigue a Zuloaga y Morrone (1999).
Para determinar la fenología de floración de cada una de las especies estudiadas, en cada visita se registró la presencia/ausencia de flores sobre 10 plantas marcadas de cada especie distribuidas al azar sobre transectas preestablecidas descriptas en Medan et. al (2006). La fenología de floración obtenida fue comparada con datos existentes en la bibliografía (Murrielo et al. 1993).

La tasa de fructificación natural y el modo de dispersión del polen de las especies estudiadas se evaluaron mediante los siguientes tratamientos: (1) polinización controlada; (2) captura de insectos con trampas para determinar el transporte de polen entomófilo (3) captura de polen anemófilo mediante colectores gravimétricos.

\section{Tratamientos de polinización controla-} da: se tomaron al azar individuos de las cuatro especies arbóreas $(\mathrm{n}=8,15,16$ y 15 , para $C$. tala, J. rhombifolia, S. longifolia y S. buxifolia, respectivamente) y se marcaron tres ramas en cada árbol. En cada rama se realizó uno de los siguientes tratamientos: (a) autopolinización espontánea, las ramas fueron encerradas con bolsas de tela que impiden el flujo de polen exógeno, (b) polinización anemófila, las ramas marcadas fueron embolsada con tul, que permitió el paso del polen anemófilo y excluyó a los visitantes florales, y (c) polinización natural (testigo). Las ramas testigos estuvieron expuestas al flujo polínico natural (biótico y abiótico). Las ramas utilizadas para los tratamientos de anemofilia y autopolinización espontánea fueron embolsadas en un estadio previo a la antesis. Al inicio de la experiencia se contaron las flores hermafroditas (C. tala, $S$. buxifolia y $J$. rhombifolia) y femeninas $(S$. longifolia) en cada rama. En los tratamientos de anemofilia y autopolinización espontánea se retiraron, con pinzas de punta fina las flores ya abiertas. Al finalizar la experiencia se contaron los frutos producidos en las ramas de cada tratamiento, y se calculó la relación fruto/flor para cada especie bajo los tres tratamientos. Se comparó la proporción fruto/flor obtenida en cada tratamiento con el test no paramétrico 
de Kruskal-Wallis para cada especie arbórea. Para las especies donde se hallaron diferencias significativas entre los tres tratamientos se realizaron comparaciones múltiples no paramétricas a posteriori, prueba de Dunn, corregidas por empates (Daniels 1990).

Cargas polínicas de visitantes florales: para examinar las cargas polínicas en los visitantes florales se colocaron periódicamente tres trampas para insectos (recipientes amarillos de $23 \mathrm{~cm}$ de diámetro, con agua azucarada y gotas de detergente). Las trampas se instalaron por la mañana y se recolectaron por la tarde de cada día de muestreo. En el laboratorio se retiraron los insectos del líquido de las trampas, observando que las cargas polínicas no se continuaban adheridas al cuerpo. Para examinar el polen obtenido de las trampas para insectos, el líquido de las mismas se centrifugó a 3500 rpm, durante 15 minutos, montándose luego el sedimento en glicerina-gelatina coloreada con safranina. Posteriormente se determinó, bajo microscopio, la identidad taxonómica de los granos de polen al mayor nivel posible comparándolos con una colección polínica de referencia formada con las plantas de la comunidad y con la palinoteca del Laboratorio de Paleobotánica y Palinología de la Facultad de Ciencias Exactas y Naturales de la Universidad de Buenos Aires. Se contó la cantidad de granos de polen de las especies focales y los granos de polen de los otros taxa fueron agrupado en "Otras especies" (Cuadros 2 y 3). Posteriormente, los insectos capturados en las trampas fueron asignados a morfo-especies y una muestra fue montada y determinada al mayor nivel taxonómico posible, en algunos casos con ayuda de especialistas (ver Agradecimientos). Las morfoespecies capturadas fueron comparadas con las reportadas por Basilio \& Torretta (2006) como visitantes florales de las especies focales en el mismo sitio de estudio.

Captura de polen anemófilo: para estudiar el polen transportado por el viento se colocaron tres captadores aeropalinológicos tipo Tauber (Faegri y Iversen 1989) entre cordones de bosque, en zona abierta y adosados a un poste a un metro del suelo (en las mismas condiciones). Los mismos fueron colocados desde julio a noviembre, coincidiendo con la época de floración de las especies focales. Los captadores aeropalinológicos fueron colectados mensualmente (en agosto se retiraron a mediados de mes, agrupando los datos de julio con la primer quincena de agosto [julio-agosto] y la segunda quincena de agosto con los datos de septiembre [agosto-septiembre]). El líquido contenido en los tres captadores se unificó para formar una muestra en cada período. El contenido polínico se concentró por centrifugado durante 15 minutos a $3500 \mathrm{rpm}$ y luego se acetolizó según la técnica de Erdtman (Faegri y Iversen 1989). El sedimento se montó en gelatina glicerina con safranina y se identificó por comparación con la colección polínica de referencia antes mencionada.

\section{RESULTADOS}

La fenología de floración de las cuatro especies estudiadas se presenta en la Figura 1.

La proporción de fruto/flor no difirió significativamente entre los tres tratamientos en Celtis tala $(\mathrm{H}=1.420, \mathrm{p}=.4916) \mathrm{ni}$ en Jodina rhombifolia $(\mathrm{H}=3.853, \mathrm{p}=.1456)$. En el caso de Scutia buxifolia $(\mathrm{H}=7.591, \mathrm{p}=.0225)$ y de Schinus longifolia $(\mathrm{H}=13.139, \mathrm{p}=.0014)$ se hallaron diferencias significativas entre los tres tratamientos (Cuadro 1).

El polen recuperado de las trampas para insectos mostró gran variación entre los cuatro meses de estudio (Cuadro 2). En agosto y septiembre, J. rhombifolia fue la única especie focal representada en la muestra polínica de las trampas, con el $31,4 \%$ y el $8,6 \%$ respectivamente, de los granos de polen presentes. Los granos de polen de C. tala representaron una bajísima proporción. Durante octubre, el $14,8 \%$ de los granos de polen pertenecían a $S$. longifolia, mientras que $S$. buxifolia y J. rhombifolia aparecieron en bajos valores (Cuadro 2). Finalmente, el noviembre se observó una disminución en la proporción de granos de polen de S. longifolia y un aumento en los de 
Especie

1999
may jun jul ago sep oct nov dic

\section{Celtis tala}

Jodina rhombifolia

Schinus longifolia

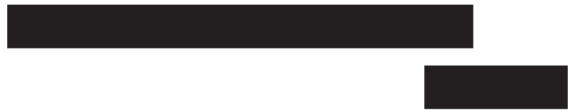

Scutia buxifolia

Fig. 1. Fenología de floración de las cuatro especies arbóreas estudiadas en el campo Carretero del Parque Costero del Sur, durante 1999.

Fig. 1. Flowering phenology of the four arboreal species studied in the Carretero field located in the Parque Costero del Sur, during 1999.

\section{CUADRO 1}

Proporción de frutos/flores (media \pm desvio estándar) de las cuatro especies arbóreas estudiadas en el campo Carretero del Parque Costero del Sur, durante 1999.

Letras iguales indican diferencias no significativas $(P=0,05)$

TABLE 1

Rate of fruits/flowers (mean \pm standard deviation) of the four arboreal species studied in the Carretero field in the Parque Costero del Sur, during 1999.

Same letters are not significantly different $(P=0,05)$

\begin{tabular}{|c|c|c|c|}
\hline Especie & $\begin{array}{c}\text { Autopolinización } \\
\text { espontánea }\end{array}$ & $\begin{array}{l}\text { Polinización } \\
\text { anemófila }\end{array}$ & $\begin{array}{c}\text { Polinización } \\
\text { libre }\end{array}$ \\
\hline Celtis tala & $0.70 \pm 0.22 \mathrm{a}$ & $0.75 \pm 0.18 \mathrm{a}$ & $0.60 \pm 0.26 \mathrm{a}$ \\
\hline Jodina rhombifolia & $0.04 \pm 0.05 \mathrm{a}$ & $0.14 \pm 0.16 \mathrm{a}$ & $0.05 \pm 0.04 \mathrm{a}$ \\
\hline Schinus longifolia & $0.06 \pm 0.16 \mathrm{a}$ & $0.29 \pm 0.19 b$ & $0.34 \pm 0.22 b$ \\
\hline Scutia buxifolia & $0.02 \pm 0.03 \mathrm{a}$ & $0.02 \pm 0.05 \mathrm{a}$ & $0.07 \pm 0.07 \mathrm{~b}$ \\
\hline
\end{tabular}

\section{CUADRO 2}

Porcentaje de polen transportado por insectos capturados en trampas en el campo Carretero del Parque Costero del Sur, desde agosto a noviembre de 1999. Ago = agosto, Sep = septiembre, Oct = octubre y Nov = noviembre

TABLE 2

Pollen transported by insects captured in traps in the Carretero field in the Parque Costero del Sur, from August to November, 1999. Ago = August, Sep $=$ September, Oct $=$ Octuber and Nov $=$ November

$\begin{array}{lcccc}\text { Especies } & \text { Ago } & \text { Sep } & \text { Oct } & \text { Nov } \\ \text { Cetis tala } & 0.3 & 0.8 & 0.0 & 0.0 \\ \text { Jodina rhombifolia } & 31.4 & 8.6 & 0.7 & 0.0 \\ \text { Schinus longifolia } & 0.0 & 0.0 & 14.8 & 3.6 \\ \text { Scutia buxifolia } & 0.0 & 0.0 & 0.2 & 3.0 \\ \text { Otras especies } & 68.3 & 90.6 & 84.3 & 93.4 \\ & 100.0 & 100.0 & 100.0 & 100.0\end{array}$


S. buxifolia respecto al mes anterior. En los cuatros meses relevados la proporción de polen de "otros taxa" fue mayor que la proporción de cualquiera de las especies focales. En las trampas para visitantes florales, se recolectaron aproximadamente 170 morfo-especies de insectos, entre los cuales se encontraban todos los visitantes de las cuatro especies estudiadas (Basilio \& Torretta 2006).

El polen anemófilo colectado por los captadores aeropalinológicos (tipo Tauber), también exhibió variación entre meses, aunque estuvo representado por menor cantidad de taxa (Cuadro 3). La única especie representada durante todo el muestreo fue Celtis tala, con el $2.0,8.1,85.9$ y $31.5 \%$ respectivamente para los cuatro períodos estudiados (Cuadro 3). Las demás especies focales estuvieron poco representadas en los distintos meses. Los granos de polen de "otros taxa" estuvieron presentes durante todos los períodos analizados y, excepto en octubre, su representación fue mayor que la de las especies focales (Cuadro 3).

\section{DISCUSIÓN}

En este trabajo se abordó la dispersión polínica en forma integral. Si bien los métodos de captura de polen en ambas vías de dispersión (biótica y abiótica) no permiten comparar valores absolutos de cantidad o volumen de polen, la información obtenida se complementa y contribuye a la descripción del comportamiento polínico de los árboles de este bosque. Aunque no se realizaron cruzamientos dirigidos, los resultados de los tratamientos permiten inferir el sistema reproductivo de estas especies.

Celtis tala resultó ser marcadamente anemófila. Si bien C. tala es morfológicamente una especie andromonoica, las flores hermafroditas podrían comportarse como femeninas como ya fue mencionado para C. iguanaea (Jacq.) Sarg. (Vaz de Arruda \& Sazima 1988), por lo cual la especie sería monoica debido a que tendría flores funcionalmente unisexuales. Bawa et al. (1985) mencionan monoecia en Celtis schippii Trel. Ex Wendl., en cambio, Bullock (1985) menciona a C. iguanaea (Jacq.) Sarg. y $C$. caudata Planch. como especies polígamomonoicas. La morfología floral, polínica, la presencia de flores "unisexuales" y la floración previa a la foliación, al igual que una alta relación polen/óvulo, son características propias de este tipo de dispersión polínica (Faegri \& van der Pijl 1979). El polen de esta planta estuvo muy representado en los colectores aeropalinológicos durante el período de floración y muy poco en las trampas para insectos, aunque en las mieles estudiadas de los bosques del norte del país, el polen de Celtis tala se encuentra frecuentemente (Basilio y Noetinger 2000, Salgado 2006, Cabrera y Salgado 2006). Vaz de

\section{CUADRO 3}

Polen aerotransportado capturado en colectores aeropalinológicos tipo Tauber en el campo Carretero del Parque Costero del Sur, durante 1999. Ago = agosto, Sep = septiembre, Oct = octubre y Nov = noviembre

TABLE 3

Air-transported pollen captured in Tauber type air-palinological collectors in the Carretero field in the Parque Costero del Sur, during 1999. Ago = August, Sep $=$ September, Oct $=$ Octuber y Nov $=$ November

$\begin{array}{lcccc}\text { Especies } & \text { Jul-Ago } & \text { Ago-Sep } & \text { Oct } & \text { Nov } \\ \text { Celtis tala } & 2.0 & 8.1 & 85.9 & 31.5 \\ \text { Jodina rhombifolia } & 0.0 & 2.7 & 0.0 & 0.0 \\ \text { Schinus longifolia } & 0.0 & 0.0 & 3.0 & 0.0 \\ \text { Scutia buxifolia } & 0.0 & 6.8 & 3.0 & 0.8 \\ \text { Otras especies } & 98.0 & 82.4 & 8.1 & 67.7 \\ & 100.0 & 100.0 & 100.0 & 100.0\end{array}$


Arruda \& Sazima (1988) señalan la presencia de abejas (principalemente Apis mellifera L.) colectando polen en flores de $C$. iguanaea. Basilio \& Torretta (2006) solamente mencionan dos especies de insectos visitando flores de C. tala, pero no observaron abejas domésticas. La falta de diferencias significativas en los tratamientos de polinización natural y polinización con exclusión de visitantes florales confirma este hecho. Por otra parte, la falta de diferencias significativas en la formación de frutos entre tratamientos podría indicar que C. tala es una especie autocompatible. Estos resultados son congruentes con el comportamiento polínico y sistema reproductivo de otra especie de Celtis (Vaz de Arruda \& Sazima 1988) estudiada en Brasil.

El polen de Jodina rhombifolia fue encontrado en las trampas de insectos pero su representatividad en los colectores gravimétricos fue baja. La dispersión polínica de esta especie sería llevada a cabo principalmente por los visitantes florales, aunque debido a la falta de diferencias significativas entre los tratamientos de polinización, esta especie podría comportarse como autógama espontánea. J. rombhifolia florece durante el invierno, un período del año en el cual disminuye la abundancia de polinizadores (Basilio et al. 2006), por lo cual la autogamia espontánea podría favorecer a un aseguramiento reproductivo (Kalisz y Vogler 2003). El aseguramiento reproductivo por reproducción uniparental (autopolinización o asexualidad) es común cuando la polinización cruzada es impredecible, ya sea por escasez de polinizadores o individuos coespecíficos, por limitación polínica (Eckert et al. 2006 y citas allí).

En Schinus longifolia, la relación fruto/flor en el tratamiento de exclusión de polen exógeno fue significativamente menor que los otros dos tratamientos. Esta especie es dioica y sus flores unisexuales, por lo que este resultado indicaría no presencia de apomixis, de partenocarpia y la no funcionalidad del gineceo reducido de las flores masculinas. Este mismo resultado fue reportado para $S$. terebinthifolius Raddi (Lenzi \& Orth 2004). Si bien S. longifolia mantiene un abundante elenco de visitantes florales (Basilio y Torretta 2006), no se hallaron diferencias significativas entre los tratamientos de polinización libre y anemofilia, por lo que podría tratarse de una especie ambófila. El registro de granos de polen de $S$. longifolia en las cargas polínicas recuperadas de las trampas de insectos (14.8\% de la carga total de octubre) apoyan la posibilidad de ambofilia en esta especie, aunque como mencionan Bronstein et al. (2006) la visita de los polinizadores podría ser desigual a las plantas masculinas y femeninas, debido a la diferencia de recompensas (polen y néctar en plantas masculinas y solo néctar en las femeninas). Por otro lado, la baja abundancia relativa de polen de $S$. longifolia en los colectores gravimétricos $(3.0 \%)$ podría deberse a que, de las especies estudiadas, es la que posee una menor abundancia en la comunidad y a que la producción polínica en esta especie es menor que la de $C$. tala, y debido que las floraciones de ambas especies se superponen, el porcentaje relativo encontrado estaría subestimando la disponibilidad de su polen en la atmósfera de la comunidad. En S. longifolia, una especie con flores unisexuales, este sistema dual podría ser el resultado de un mecanismo de flexibilidad del sistema de polinización o una transición evolutiva (entre polinización biótica/abiótica) como sugieren Peeters y Totland (1999) para especies del género Salix (ver Culley et al. 2002 para otras especies). Schinus longifolia podría estar ubicada en un paso intermedio en relación con la dioecia típica de las plantas anemófilas (Peeters y Totland 1999, Wallander 2001) y la monoecia ancestral de las plantas primariamente entomófilas. Por otra parte, el bosque donde se llevó a cabo este estudio está próximo al límite sur de la distribución de $S$. longifolia, por lo que el comportamiento de esta población podría resultar poco representativo de la especie por las distintas relaciones interespecificas dentro de la comunidad, como también por condiciones climáticas diferentes. El estudio de varias poblaciones dentro del ámbito de distribución permitiría comprender cual/es son los factores que favorecen la ambofilia en esta especie en el talar y detectar posibles cambios evolutivos, tal como destaca 
Thompson (2005) dentro de un mosaico geográfico de adaptaciones.

Las flores de $S$. buxifolia, visitadas por numerosas especies de insectos (Basilio y Torretta 2006), requieren el servicio de polinizadores para la formación de frutos. La relación fruto/flor obtenida mediante tratamiento control, que permitió el acceso a los visitantes florales, fue significativamente mayor que en los otros dos tratamientos, aunque los valores fueron muy bajos. La baja (o nula) formación de frutos en los tratamientos de polinización anemófila y autopolinización espontánea indican que la especie sería autoincompatible. Este resultado concuerda con la autoincompatibilidad demostrada para numerosas especies de Rhamnaceae (Webb 1985, Medan 1993, Medan \& Arce 1999, Medan \& Basilio 2001)

La dispersión polínica y el sistema reproductivo difirieron entre las cuatro especies estudiadas. Las posibilidades de autogamia y polinización abiótica podrían indicar a Celtis tala como la especie más tolerante a disturbios en su reproducción. En el caso de Jodina rhombifolia, el aseguramiento reproductivo a través de la autopolinización espontánea permitiría la reproducción de esta especie en condiciones de falta polinizadores. Scutia buxifolia, debido a la autoincompatibilidad y a la necesidad de polinizadores bióticos y Schinus longifolia, debido a la dioecia y a la actual escasez de ejemplares, podrían ser más sensibles a los disturbios y a los factores que limiten su reproducción.

\section{AGRADECIMIENTOS}

A los dueños de la Estancia Carretero por permitirnos llevar a cabo el trabajo de campo dentro de su propiedad. Al Laboratorio de Paleobotánica y Palinología de la Facultad de Ciencias Exactas y Naturales de la Universidad de Buenos Aires, donde se procesaron las muestras. Roig Alsina y C. Thompson colaboraron en la identificación de algunos insectos. Agradecemos a J. Pensiero por la lectura crítica de una versión preliminar y a dos revisores anónimos que con sus comentarios enriquecieron el trabajo.

\section{RESUMEN}

Los "talares" del E de la provincia de Buenos Aires son bosques xerófitos costeros estructurados por pocas especies arbóreas rodeados de una matriz de suelos más bajos y húmedos. Estudiamos los parámetros reproductivos de las especies arbóreas más representativas (Celtis tala, Scutia buxifolia, Jodina rhombifolia y Schinus longifolia). La dispersión polínica fue estudiada a través de trampas para visitantes florales (dispersión biótica) y utilizando recolectores gravimétricos de polen (dispersión abiótica). El éxito reproductivo (tasa de formación de frutos) de las especies focales fue estudiado a través de embolsado de flores con bolsas de distintos tipos de malla. El sistema reproductivo varió entre las especies. C. tala resultó anemófila y autocompatible, S. buxifolia fue entomófila y dependiente de los visitantes florales. J. rhombifolia fue una especie entomófila, aunque la autogamia espontánea podría favorecer al aseguramiento reproductivo en caso de falta de polinizadores. Finalmente, S. longifolia podría ser una especie ambófila (polinizada por insectos y por el viento). Este sistema dual podría ser el resultado de un mecanismo de flexibilidad del sistema de polinización o una transición evolutiva.

Palabras clave: dispersión polínica, especies arbóreas, polinización, anemofilia, ambofilia, zoofilia, bosque xerófito.

\section{BIBLIOGRAFÍA}

Arturi, M.F., C.A. Pérez, M. Horlent, J.F. Goya \& S. Torres. 2006. El manejo de los talares de Magdalena y Punta Indio como estrategia para su conservación, p. 37-45. In E. Mérida \& J. Athor (eds.). Talares bonaerenses y su conservación. Fundación de Historia Natural "Félix de Azara", Buenos Aires, Argentina.

Basilio, A.M.\& M. Noetinger. 2000. Análisis polínico de mieles de la Región Chaqueña: comparación del origen floral entre zonas: domo central y esteros, cañadas y selvas de ribera. RIA 31: 127-134.

Basilio, A.M., D. Medan, J.P. Torretta \& N. Bartoloni. 2006. A year-long plant-pollinator network. Austral Ecol. 31: 975-983.

Basilio, A.M. \& J.P. Torretta. 2006. Polinización en el talar de Magdalena, p. 106-116. In E. Mérida \& J. Athor (eds.). Talares bonaerenses y su conservación. Fundación de Historia Natural "Félix de Azara", Buenos Aires, Argentina.

Bronstein, J.L, R. Alarcón, M. Geber. 2006. The evolution of plant insect mutualisms. New Phytol. 172: 412-428. 
Bullock, S.H. 1985. Breeding systems in the flora of a tropical deciduous forest in México. Biotropica 17: 287-301.

Bullock, S.H. 1994. Wind pollination of neotropical dioecious trees. Biotropica 26: 172-179.

Bush, M.B. \& R. Rivera. 2001. Reproductive ecology and pollen representation among neotropical trees. Global Ecol. Biogeogr. 10: 359-367.

Cabrera, M.M. \& C.R. Salgado. 2006. Contribución al estudio de la flora melífera de la provincia de Formosa, Argentina. Comunicaciones Científicas y Tecnológicas, Universidad Nacional del Nordeste, Corrientes, Argentina.

Chichizola, S. 1993. Las comunidades vegetales de la Reserva Natural Estricta Otamendi. Parodiana 8: 227-263.

Culley, T.M., S.G.Weller \& A.K. Sakai. 2002. The evolution of wind pollination in

Angiosperms. Trends Ecol. Evol. 17: 361-369.

Daniels, W.W. 1990. Applied nonparametric statistics. PWS-Kent, Boston, EEUU. 635 p.

Eckert, C.G., K.E. Samis \& S. Dart. 2006. Reproductive assurance and the evolution of uniparental reproduction in flowering plants, p. 183-203. In L.D. Harder \& S.C.H. Barrett (eds.). Ecology and evolution of flowers. Oxford University, Oxford, England.

Endress, P.K. 1998. Diversity and evolutionary biology of tropical flowers. Cambridge University, Cambridge, England.

Fabris, H. 1965. Anacardiaceae. In A.L. Cabrera (ed.). Flora de la provincia de Buenos Aires. Colecc. Ci. Inst. Nac. Tecnol. Agropecu. 4: 130-134.

Faegri, K. \& J. Iversen. 1989. Textbook of Pollen Analysis. Wiley, Nueva York, New York, EEUU.

Faegri, K. \& L. van der Pijl. 1979. The principles of pollination ecology. Pergamon, Oxford, England.

Goya, J.F., G. Placci, M.F. Arturi \& A. Brown. 1992. Distribución y características estructurales de los talares de la reserva de la biosfera "Parque Costero del Sur”. Rev. Fac. Agr. (La Plata) 68: 53-64.

Hunziker, A.T. \& N.M. Dottori. 1976. Contribución al conocimiento sobre los talas (Celtis, Ulmaceae) de Argentina, con especial referencia a la región mediterránea. Kurtziana 9: 103-140.
Johnston, M.C. 1974. Revision of Scutia (Rhamnaceae). Bull. Torrey Bot. Club 101: 64-72.

Kalisz, S. \& D.W. Vogler. 2003. Benefits of autonomous selfing under unpredictable pollinator environments. Ecology 84: 2928-2942.

Lenzi, M. \& A.I. Orth. 2004. Caracterizaçao funcional do sistema reproductivo da aroeira-vermelha (Schinus terebinthifolius Raddi), em Florianópolis-SC, Brasil. Rev. Bras. Frutic. 26: 198-201.

Medan, D. 1993. Breeding system and maternal success of a perennial hermaphrodite, Discaria americana (Rhamnaceae). New Zealand J. Bot. 31: 175-184.

Medan, D. \& A.M. Basilio. 2001. Reproductive biology of Colletia spinosissima (Rhamnaceae) in Argentina. Plant Syst. Evol. 229: 79-89.

Medan, D. \& M.E. Arce. 1999. Reproductive biology of the Andean-disjunct genus Retanilla (Rhamnaceae). Plant Syst. Evol. 218: 281-298.

Medan, D., A.M. Basilio, M. Devoto, N.J. Bartoloni, J.P. Torretta \& T. Petanidou. 2006. Measuring generalization and connectance in temperate, year-long active systems, p. 245-259. In N. Waser \& J. Ollerton (eds.). Specialization and generalization in plant-pollinator interactions. University of Chicago, Chicago, EEUU.

Morello, J. 2006. Acciones urbanas y conservación de talares: un marco de negociación, p. 16-31. In E. Mérida \& J. Athor (eds.). Talares bonaerenses y su conservación. Fundación de Historia Natural "Félix de Azara", Buenos Aires, Argentina.

Muñoz, J.D. 1990. Anacardiaceae. In R. Spichiger \& L. Ramella (eds.). Flora del Paraguay. conservatoire et Jardin Bataníques de la Ville de Gèneve and Missouri Botanical Garden, Geneva, Suiza.

Murriello, S., M. Arturi \& A. Brown. 1993. Fenología de las especies arbóreas de los talares del este de la Provincia de Buenos Aires. Ecol. Austral 3: 25-31.

Parodi, L. 1940. La distribución geográfica de los talares de la provincia de Buenos Aires. Darwiniana 4: 33-56.

Peeters, L. \& Ø. Totland. 1999. Wind to insect pollination ratios and floral traits in five alpine Salix species. Can. J. Bot. 77: 556-563.

Proctor, M., P. Yeo \& A. Lack. 1996. The natural history of pollination. Timber, Portland, Oregon, EEUU. 479 p.

Ribichich, A.M. 1996. Celtis tala Planchon (Ulmaceae s. 1.) seedling establishment on contrasting soils 
microdisturbances: a greenhouse trial concerning adults' field distribution patterns. Flora 191: 321327.

Ruiz, F. 2003. Análisis comparativo del crecimiento de una especie leñosa nativa y otra exótica de los talares de Magdalena (Pcia. de Buenos Aires, República Argentina). Tesis de licenciatura, Facultad de Ciencias Exactas y Naturales, Universidad de Buenos Aires, Buenos Aires, Argentina.

Salgado, C.R. 2006. Flora melífera en la provincia del Chaco. PROSAP. Impreso por Ministerio de la Producción del Chaco, Chaco, Argentina.

Stupino, S.A., M.F. Arturi \& J.L. Frangi. 2004. Estructura del paisaje y conservación de los bosques de Celtis tala Gill. ex Planch. del NE de la provincial de Buenos Aires. Rev. Fac. Agr. (La Plata) 105: 37-45.

Thompson, J. N. 2005. The Geographic Mosaico of Coevolution. University of Chicago, Chicago, EEUU. $443 \mathrm{p}$.

Tortosa, R. 1995. Rhamnaceae. Flora Fanerogámica Argentina 9: 1-18.
Ulibarri, E. 1987. Santalaceae, p. 101-105. In A. Burkart, N. Troncoso \& N. Bacigalupo (eds.). Flora Ilustrada de Entre Ríos (Argentina). Colecc. Ci. Inst. Tecnol. Agropecu. 6: 1001-1005.

Vaz de Arruda, V.L. \& M. Sazima. 1988. Polinização e reprodução de Celtis iguanaea (Jacq.) Sarg. (Ulmaceae), uma espécie anemófila. Rev. Brasil. Bot. 11: 113-122.

Vervoorst, F. 1967. Las comunidades vegetales de la depresión del Salado (Provincia de Buenos Aires). INTA, Buenos Aires, Argentina. 262 p.

Wallander, E. 2001. Evolution of wind-pollination in Fraxinus (Oleaceae) - an ecophylogenetic approach. Ph.D. thesis, Göteborg University, Göteborg, Sweden.

Webb, C.J. 1985. Protandry, pollination, and sek-incompatibility in Discaria toumatou. New Zealand J. Bot. 23: 331-335.

Zuloaga, F.O. \& O. Morrone. 1999. Catálogo de las plantas vasculares de la República Argentina. II. Dicotyledoneae. Monogr. Syst. Bot. Missouri Bot. Gard. 74: 1-1269. 